\title{
Evaluation of selected parameters of the antioxidative system in patients with type 2 diabetes in different periods of metabolic compensation
}

\section{Jacek Rysz¹, Robert Błaszczak ${ }^{1}$, Maciej Banach², Kornelia Kędziora-Kornatowska ${ }^{3}$, Tomasz Kornatowski ${ }^{4}$, Wojciech Tański ${ }^{5}$ and Józef Kędziora ${ }^{6}$}

1 2nd Department of Family Medicine, University Hospital No. 2, Medical University of Łódź, Poland

2 Department of Cardiology, 1st Chair of Cardiology and Cardiac Surgery, University Hospital No. 3, Medical University of Łódź, Poland

3 Department and Clinic of Geriatrics, Medical Academy of Bydgoszcz, Poland

${ }^{4}$ Department of Pharmacology and Therapy, Medical Academy of Bydgoszcz, Poland

5 Department of Internal Medicine, Military Hospital of Wrocław, Poland

${ }^{6}$ Department of Clinical Chemistry and Biochemistry, Medical University of Łódź, Poland

Received: 2006.10.19, Accepted: 2007.04.02, Published online first: 2007.10.01

\begin{abstract}
Introduction: The aim of the study was to evaluate some selected parameters of the antioxidative system in patients with type 2 diabetes.

Materials and Methods: Eighty-one type 2 diabetes patients took part in the study (39 patients with metabolically balanced and 42 with metabolically unbalanced diabetes). The control group consisted of 30 healthy people. The total antioxidant capacity of plasma was measured fluorometrically using phycoerythrin. To calculate the low-molecular-weight antioxidant concentration in the plasma samples, the duration of Trolox activity as a function of its concentration in the sample was measured. The activity of antioxidative enzymes in red blood cells was determined using the Misra and Fridovich method and Beers and Sizer method. Results: The total plasma antioxidant capacity and the low-molecular-weight antioxidant concentration in the group of patients with metabolically compensated type 2 diabetes were statistically significantly higher than in the group of patients with metabolically uncontrolled diabetes. The activity of antioxidative enzymes was found to be higher in the group of type 2 diabetes patients at the stage of metabolic balance.
\end{abstract}

Conclusions: The obtained results confirm the thesis of glucose toxicity and intensification of oxidative stress in patients with diabetes.

Key words: oxidative stress, diabetes mellitus, antioxidative enzyme.

Corresponding author: Jacek Rysz, M.D. PhD., 2nd Department of Family Medicine, Medical University of Łódź, Żeromskiego 113, 90-549 Łódź, Poland, tel./fax: +48 42 639-34-65, e-mail: jacek.rysz@skwam.lodz.pl

\section{INTRODUCTION}

Oxidative stress is a state characterized by increased activity of reactive oxygen species (ROS). Its development is a consequence of a pro-oxidative disturbance in the oxidation-reduction balance [18, 38, 39]. Although the human body is capable of free radical inactivation under physiological conditions, a major balance upset in the system of oxidants and antioxidants leads to a breakdown of cell and tissue integrity and the chemical modification of proteins, nucleic acids, lipids, and carbohydrates [2, 20, 23, 33, 37].

The effects of ROS influence may be varied. They lead, among other things, to the oxidation of low-mole- cular-weight compounds (glutathione, nicotinamideadenine nucleotides), collagen degradation, hyaluronic acid depolymerization, hemoglobin oxidation, protein transport, and enzyme inactivation [4, 5, 19, 34]. ROS are also responsible for DNA strand breaks, chromosome damage, membrane lipid peroxidation, inhibition of oxidative phosphorylation in mitochondria, perturbation of intracellular $\mathrm{Ca}^{2+}$ homeostasis, platelet aggregation, and lipid peroxidation [30].

In the course of evolution, organisms have developed some more or less refined mechanisms protecting them against the harmful activity of free radicals, both in enzymatic and non-enzymatic ways $[1,13,30,32]$. These 
include a number of enzymes which directly catalyze reactions involving ROS, such as superoxide dismutase (SOD), glutathione peroxidase, and catalase (CAT), as well as enzymes which catalyze these reactions indirectly, e.g. glutathione transferase and glucose-6-phosphate dehydrogenase. Low-molecular-weight compounds, defined as low-molecular-weight antioxidants, also play an important role in maintaining the oxidation-reduction balance. The most important low-molecular-weight antioxidants are glutathione, ascorbate, and vitamin E. A number of other compounds, such as cysteine, uric acid, bilirubin, and catecholamines, are also known antioxidants [1, 30, 32]. Although extracellular fluids do not show much antioxidant capacity, they contain both antioxidative enzymes and low-molecular-weight antioxidants. The activity of antioxidative enzymes in plasma is low compared with their intracellular activity, but plasma contains a number of low-molecular-weight antioxidants, the most important of which is ascorbate, which reacts with superoxide anion radical, hydrogen peroxide, peroxide radicals, and singlet oxygen. Other antioxidants are, for example, tocopherols, carotenoids, and uric acid.

Growing attention has been paid to the participation of ROS in the pathomechanisms of many diseases, including diabetes. It was observed that in the course of diabetes an intensification of oxidative stress takes place, with considerable predominance of oxidative factors over antioxidative mechanisms [30]. As in other diseases, oxidative stress in diabetes is a result of increased ROS production on the one hand and decreased antioxidant system activity on the other. The evaluation of antioxidative enzyme activity in this disease is controversial. In experimental studies, both declines in antioxidative enzyme activity, e.g. glutathione peroxidase, and increases in the activities of other key cellular antioxidative enzymes, i.e. SOD and CAT in kidney of rats with streptozotocin-induced diabetes, were described [4, 21, 22]. In red blood cells from patients with diabetes, an increased amount of the glycated form of SOD accompanied by a lower activity of this enzyme [27, 29] was observed. It was shown in some experimental and clinical studies that intensification of ROS production depends not only on the degree of diabetes compensation, but also on its duration [3]. The aim of this study was therefore to evaluate some selected parameters of the antioxidative system in patients with type 2 diabetes, both controlled and uncontrolled, by determining the activity levels of antioxidative enzymes such as SOD and CAT in the patients' red blood cells and the total antioxidant capacity and concentration of low-molecular-weight antioxidants in plasma.

\section{MATERIALS AND METHODS}

\section{Patients population}

Eighty-one patients with diabetes mellitus (28 women [34.6\%], 53 men [65.4\%]) with a mean age of $63.4 \pm 9.7$ years were included to the study. The mean duration of the disease was over $14 \pm 1.2$ years. Because of difficulties in choosing suitable people for the control group comparable in terms of age and sex to the examined patients and not taking drugs with known antioxidative properties or drugs potentially influencing cellular metabolism, a reference group (C) consisting of 30 healthy people (mean age: $47.1 \pm 8.2$ years), who were a representative group for the given region, was formed. Permission to carry out the study was granted by the Ethics Committee of Medical University of Łódź, Poland (No. 139/01).

All the participants of the study underwent a subjective and an objective examination and they also had their blood tested with respect to blood glucose level, total cholesterol, triglycerides, plasma creatinine, and glycosylated hemoglobin level. All the examined persons had their body mass index calculated. The precondition for participation in the study was not taking drugs with recognized antioxidative properties. The participants were non-smokers and were also on a diabetic diet excluding foods with recognized antioxidative properties. Patients qualified for the study were consulted by a nephrologist, an ophthalmologist, and a vascular surgeon to exclude micro- and macroangiopathy (Table 1).

Table 1. The clinical characteristics of investigated patients

\begin{tabular}{lccc}
\hline & $\mathrm{DM}_{\mathrm{C}}$ & $\mathrm{DM}_{\mathrm{U}}$ & $\mathrm{C}$ \\
\hline Number of patients & 39 & 42 & 30 \\
Mean age $(\mathrm{x} \pm \mathrm{SD})$ & $64.3 \pm 9.8$ & $62.7 \pm 10.2$ & $47.1 \pm 8.2$ \\
$\mathrm{HbA}_{1} \mathrm{c}(\%)$ & $6.7 \pm 1.3$ & $8.0^{\mathrm{a}} \pm 1.7$ & $5.7 \pm 1.1$ \\
$\mathrm{BMI}\left(\mathrm{kg} / \mathrm{m}^{2}\right)$ & $23.8 \pm 3.7$ & $25.4 \pm 3.8$ & $21.9 \pm 2.8$ \\
Total cholesterol $(\mathrm{mmol} / \mathrm{l})$ & $5.8 \pm 1.4$ & $6.5^{\mathrm{b}} \pm 1.4$ & $4.5 \pm 1.1$ \\
LDL-cholesterol $(\mathrm{mmol} / \mathrm{l})$ & $2.18 \pm 1.1$ & $3.11^{\mathrm{b}} \pm 1.3$ & $2.13 \pm 0.9$ \\
HDL-cholesterol $(\mathrm{mmol} / \mathrm{l})$ & $1.41 \pm 0.6$ & $1.21^{\mathrm{b}} \pm 1.1$ & $1.36 \pm 1.2$ \\
Triglyceride $(\mathrm{mmol} / \mathrm{l})$ & $1.8 \pm 0.7$ & $2.4^{\mathrm{b}} \pm 1.2$ & $1.2 \pm 0.4$ \\
Glucose $(\mathrm{mmol} / \mathrm{l})$ & $7.8 \pm 1.7$ & $9.3^{\mathrm{a}} \pm 1.5$ & $4.8 \pm 1.5$ \\
Creatynine $(\mu \mathrm{mol} / \mathrm{l})$ & $88.5 \pm 20.3$ & $89.1^{\mathrm{b}} \pm 23.4$ & $86.7 \pm 14$ \\
Albumin secretion $(\mathrm{mg} / 24 \mathrm{~h})$ & $10.8 \pm 2.6$ & $11.7^{\mathrm{b}} \pm 2.5$ & $8.3 \pm 1.9$ \\
\hline
\end{tabular}

$\mathrm{DM}_{\mathrm{C}}$ - patients with metabolically compensated type 2 diabetes, $\mathrm{DM}_{\mathrm{U}}$ - patients with metabolically uncontrolled type 2 diabetes, $\mathrm{C}$ - control, $\mathrm{BMI}$ - body mass index. ${ }^{\mathrm{a}} \mathrm{p}<0.05 \mathrm{C}$ vs. $\mathrm{DM}_{\mathrm{U}}, \mathrm{DM}_{\mathrm{C}},{ }^{\mathrm{b}} \mathrm{p}>0.05 \mathrm{C}$ vs. $\mathrm{DM}_{\mathrm{U}}, \mathrm{DM}_{\mathrm{C}}$. 
The fundamental criterion for inclusion in the study was diagnosed and documented type 2 diabetes. The examined patients were divided into two groups on the basis of the criteria of diabetes compensation formulated by the European Association for the Study of Diabetes: patients with metabolically compensated type 2 diabetes and patients with metabolically uncontrolled type 2 diabetes (Table 1 ).

\section{Experimental procedures}

The blood samples for the tests were collected (after an overnight fast, before the administration of morning hypoglycemic drugs) in heparin tubes from the basilic vein (250 units heparin/1 ml blood) and were then centrifuged $\left(1400 \times \mathrm{g}, 4^{\circ} \mathrm{C}, 10 \mathrm{~min}\right)$. The total antioxidant capacity and low-molecular-weight antioxidant concentration were determined in the resulting plasma. The total antioxidant capacity of plasma was measured fluorometrically using phycoerythrin [17]. To calculate the low-molecular-weight antioxidant concentration in a plasma sample, the duration of Trolox activity as a function of its concentration in the sample was measured. The activity of SOD in red blood cells was evaluated by the method of Misra and Fridovich [29]. The activity of the enzyme was determined based on the inhibition of the adrenaline peroxidation reaction by dismutase in which the superoxide anion radical takes part. The activity of CAT was evaluated by the method of Beers and Sizer [6], based on measurement of the rate of hydrogen peroxide decomposition caused by this enzyme.

\section{Statistical analysis}

As the number of persons was different in each group and deviation of the examined variables from the normal distribution was found using the Leven and Kolmogorov-Smirnoff tests, comparisons of the variables were based on non-parametric tests, mainly of the median (Mann-Whitney-U, Kruskall-Wallis ANOVA). A value of $p<0.05$ was taken to represent a statistical significance level for all the tests and analyses.

\section{RESULTS}

The concentration of glycated hemoglobin was statistically significantly higher in the group of patients with metabolically uncontrolled type 2 diabetes than in the control group and the group of patients with metabolically compensated type 2 diabetes (Table 1 ).

In both groups of patients with diabetes, both the total antioxidant capacity of plasma and low-molecularweight antioxidant concentration were statistically significantly lower compared with the reference group $(\mathrm{p}<0.05)$. Total plasma antioxidant capacity and lowmolecular-weight antioxidant concentration in the patients with metabolically compensated type 2 diabetes
Table 2. The total antioxidant capacity of plasma ( $\mathrm{min})$

\begin{tabular}{lccccc}
\hline & Mean & Median & Minimal & Maximal & SD \\
\hline $\mathrm{C}$ & 16.5 & 12.9 & 10.5 & 18.5 & 1.70 \\
$\mathrm{DM}_{\mathrm{C}}$ & $12.5^{\mathrm{a}}$ & 10.2 & 9.5 & 13.0 & 1.61 \\
$\mathrm{DM}_{\mathrm{U}}$ & $9.0^{\mathrm{a}, \mathrm{b}}$ & 8.3 & 6.5 & 10.0 & 0.86 \\
\hline
\end{tabular}

${ }^{\mathrm{a}} \mathrm{p}<0.05 \mathrm{C}$ vs. $\mathrm{DM}_{\mathrm{C}} \mathrm{DM}_{\mathrm{U}},{ }^{\mathrm{b}} \mathrm{p}<0.05 \mathrm{DM}_{\mathrm{C}}$ vs. $\mathrm{DM}_{\mathrm{U}}$.

Table 3. The concentration of low-molecular-weight antioxidants in plasma $(\mu \mathrm{mol} / \mathrm{ml})$

\begin{tabular}{lccccc}
\hline & Mean & Median & Minimal & Maximal & SD \\
\hline $\mathrm{C}$ & 687.3 & 642.4 & 594.4 & 842.3 & 84.4 \\
$\mathrm{DM}_{\mathrm{C}}$ & $584.4^{\mathrm{a}}$ & 542.3 & 487.0 & 671.3 & 71.1 \\
$\mathrm{DM}_{\mathrm{U}}$ & $468.2^{\text {a, b }}$ & 398.1 & 324.3 & 521.2 & 63.0 \\
\hline
\end{tabular}

${ }^{\mathrm{a}} \mathrm{p}<0.05 \mathrm{C}$ vs. $\mathrm{DM}_{\mathrm{C}}, \mathrm{DM}_{\mathrm{U}},{ }^{\mathrm{b}} \mathrm{p}<0.05 \mathrm{DM}_{\mathrm{C}}$ vs. $\mathrm{DM}_{\mathrm{U}}$.

were statistically significantly higher than in the patients with metabolically uncontrolled diabetes $(\mathrm{p}<0.05$; Tables 2 and 3).

Comparing the activity of SOD in the two groups of patients with type 2 diabetes, higher activity of the enzyme was observed in the patients with type 2 diabetes in the period of metabolic compensation. The differences between the activity in this group of patients and the group with diabetes in the period of metabolic decompensation were statistically significant $(\mathrm{p}<0.05)$. Comparing the values of SOD activity in the groups of patients with type 2 diabetes with the reference group, a statistically significant decrease in activity $(\mathrm{p}<0.05)$ was observed in the group of patients with type 2 diabetes (Table 4).

Statistically significantly higher CAT activity in the group of patients in the period of metabolic compensation than in the group with decompensation of diabetes was observed $(p<0.05$; Table 5). However, comparing the average CAT activities in both groups of patients with that in the reference group, a statistically significantly lower activity of CAT in both groups with type 2 diabetes $(\mathrm{p}<0.05)$ was observed (Table 5).

Table 4. SOD-1 activity in red blood cells (U/gHb)

\begin{tabular}{lccccc}
\hline & Mean & Median & Minimal & Maximal & SD \\
\hline $\mathrm{C}$ & 2394.2 & 2245.2 & 2134.1 & 2451.4 & 203.0 \\
$\mathrm{DM}_{\mathrm{C}}$ & $2156.0^{\mathrm{a}}$ & 2034.0 & 1985.6 & 2211.2 & 158.8 \\
$\mathrm{DM}_{\mathrm{U}}$ & $1979.8^{\mathrm{a}, \mathrm{b}}$ & 1884.4 & 1798.7 & 2054.3 & 123.1 \\
\hline
\end{tabular}

${ }^{\mathrm{a}} \mathrm{p}<0.05 \mathrm{C}$ vs. $\mathrm{DM}_{\mathrm{C}}, \mathrm{DM}_{\mathrm{U}},{ }^{\mathrm{b}} \mathrm{p}<0.05 \mathrm{DM}_{\mathrm{C}}$ vs. $\mathrm{DM}_{\mathrm{U}}$.

Table 5. CAT activity in red blood cells (U Bergmeyer/gHb)

\begin{tabular}{lccccc}
\hline & Mean & Median & Minimal & Maximal & SD \\
\hline $\mathrm{C}$ & 18.4 & 17.1 & 16.5 & 19.8 & 1.7 \\
$\mathrm{DM}_{\mathrm{C}}$ & $15.5^{\mathrm{a}}$ & 15.0 & 14.2 & 17.1 & 1.7 \\
$\mathrm{DM}_{\mathrm{U}}$ & $12.2^{\mathrm{a}, \mathrm{b}}$ & 11.1 & 10.6 & 13.4 & 1.0 \\
\hline
\end{tabular}

${ }^{\mathrm{a}} \mathrm{p}<0.05 \mathrm{C}$ vs. $\mathrm{DM}_{\mathrm{C}}, \mathrm{DM}_{\mathrm{U}},{ }^{\mathrm{b}} \mathrm{p}<0.05 \mathrm{DM}_{\mathrm{C}}$ vs. $\mathrm{DM}_{\mathrm{U}}$. 


\section{DISCUSSION}

Oxidative stress in diabetes, as in other ailments, is a result of the hyperproduction of reactive oxygen forms on the one hand and hypoactivity of the antioxidative system on the other. Long-term hyperglycemia may be an initializing factor for the systemic oxidative stress in diabetes. Ceriello's thesis about "glucose toxicity" [12] confirms this assertion. Intensified ROS generation induced under conditions of increased glucose concentration is an effect of increased activity of several independent metabolic pathways, such as monosaccharide autooxidation, non-enzymatic glycation intensification, protein kinase $\mathrm{C}(\mathrm{PKC})$ and phospholipase $\mathrm{A}_{2}$ activation, and increased activation of the intracellular polyol pathway $[6,9,15,16,24,28]$. Besides the increased production of ROS in diabetes, revealed indirectly by intensified lipid peroxidation, weakening of defensive mechanisms also upsets the oxidation-reduction balance. The systemic defense functions against oxidative damage are performed by two antioxidative systems: enzymatic and non-enzymatic. Their task is to prevent the formation of ROS as well as to scavenge existing ROS [35].

Many studies, both clinical and experimental, showed changes in the activities of enzymatic antioxidative systems in the course of diabetes. However, there are large discrepancies in evaluations of the activities of these enzymes in patients with diabetes. Both increases and decreases in antioxidative enzyme activity in animal experimental models were observed [20]. In our previous research, a decrease in the activities of key cellular antioxidative enzymes in the erythrocytes of patients with diabetic nephropathy was observed [36].

In the current study, statistically significantly higher SOD activity in the group of patients with metabolically compensated type 2 diabetes than in the patients with improper metabolic control was observed. Comparing the obtained values of SOD activity in each group of patients with type 2 diabetes in different periods of metabolic compensation to the reference group, a significant decrease in the activity of the enzyme was observed, especially in patients with improper metabolic diabetes control. A lowering of SOD activity has been reported in some previous publications. Matkovics et al. [28] were the first to suggest an association of lowered SOD activity with diabetes. Crouch et al. [16] showed that in experimental diabetes in rats, the activity of SOD in red blood cells and in the retina was decreased by $50 \%$ in comparison with healthy animals. Kawamura et al. [24] found an increased amount of the glycated form of SOD in the erythrocytes of patients with type 1 diabetes, which was connected with the lowered activity of this enzyme.

As for the second antioxidant enzyme studied, CAT, the lowest activity was also found in the group of patients with metabolically uncontrolled diabetes. Comparing the average values of CAT activity in both groups of patients to that in the reference group, lowered CAT activity in the groups of patients with meta- bolically uncontrolled type 2 diabetes was observed. This observation was confirmed in our previous experimental research, in which lowered CAT activity was observed both in erythrocytes and in kidney homogenate of rats with streptozotocin-induced diabetes [26]. The lowered CAT activity may be a result of increased superoxide anion radical production and a surCATus of hydrogen peroxide, which occurs under conditions of an increased glucose concentration in plasma. Superoxide anion radical is an inhibitor of CAT and hydrogen peroxide may be a precursor of other ROS, which may in turn initiate processes of lipid peroxidation, as was shown, among others, by Salahudeen et al. in their papers $[35,36]$. The decreased CAT activity observed in our own and other authors' research confirms that this enzyme may easily get inactivated as a result of ROS influence, especially under conditions of improper metabolic control and long-term hyperglycemia.

The hyperglycemia associated with diabetes and its complications may result in a deficiency or decline in the activity of plasmatic antioxidants, such as $\alpha$-tocopherol, uric acid, ferritin, albumin, and ascorbic acid, and in a decline in the activity of plasmatic antioxidative enzymes. Although extracellular fluids do not show high antioxidant capacity, they contain both antioxidative enzymes and low-molecular-weight antioxidants. The activity of antioxidative enzymes in plasma is low in comparison with their intracellular activity; however, plasma contains several low-molecular-weight antioxidants. One of the more important antioxidants is an ascorbate active in the watery environment of the body and reactive with superoxide anion radical, hydrogen peroxide, peroxide radicals, and singlet oxygen. It plays an important role in protecting protein thiol groups from oxidation. Other important plasmatic antioxidants are well as tocopherols are associated with lipoproteins which scavenge organic free radicals and break the reaction of lipid peroxidation. Carotenoids suppress singlet oxygen and react with organic free radicals created during the process of lipid peroxidation. Uric acid reacting with oxidants and combining with iron ions also plays an vital role here [11].

In our previous research, significant declines in the concentrations of such antioxidative vitamins as vitamin $\mathrm{C}$ and $\mathrm{E}$ in the plasma of rats with streptozotocininduced diabetes were observed. It was connected with lowered activity of cellular antioxidative enzymes and an intensification of lipid peroxidation processes in the kidneys of the examined animals. Moreover, a thickening of the basal membrane in the renal glomeruli was observed in these rats $[25,26]$. Decreased concentrations of vitamin $\mathrm{C}$ in the plasma and tissue of patients with diabetes and in animals with experimental diabetes were also observed by other researchers [36]. It was also revealed that antioxidative vitamin $\mathrm{C}$ and $\mathrm{E}$ supplementation inhibits increased TGF- $\beta$ secretion in the renal glomeruli of rats with diabetes by lowering the activity of PKC $[18,20,38]$. A lowering of oxidative stress intensi- 
ty and an improvement in the examined morphometric parameters of the renal glomeruli after 12 weeks of vitamin $\mathrm{C}$ and $\mathrm{E}$ supplementation were also observed in our previous research $[11,26]$.

In the diabetic patients of the present study, higher antioxidant capacity of plasma, expressed by the time of free radical inactivation by the antioxidant system in plasma, was observed in those with metabolically compensated diabetes. Moreover, a higher concentration of low-molecular-weight antioxidants was observed in the patients with metabolically compensated diabetes than in the group with improper metabolic control. However, comparing the total antioxidant capacity of plasma and the concentration of low molecular-weight-antioxidants in each group of patients with diabetes to the reference group, statistically significantly lower values of these parameters were found in the patient groups. Considering both our own and other authors' results, an hypothesis stating that the intensity of oxidative stress depends, among other things, on the duration of the disease as well as on the metabolic compensation may be formulated $[7,8,10,14]$. This is also confirmed by previous research in which a statistically significantly lowered activity of the key antioxidative enzymes in red blood cells of patients with type 2 diabetes was observed; the lowest activity of these enzymes was observed in patients with diabetic nephropathy.

The results of the examined parameters of the antioxidative system in diabetes show that patients with metabolically uncontrolled diabetes have reduced antioxidative enzyme activity, plasma antioxidant concentration, and total plasma antioxidant capacity. As mentioned above, diabetes is accompanied by systemic oxidative stress. Glucose, with an aldehyde group in its molecule, reacts with the encountered amino groups. As the result of this reaction the proteins undergo a non-enzymatic glycosylation, which leads to the formation of the advanced glycation endproducts. Furthermore, what has also mentioned above, the hyperactivity of the generation of ROS evoked by elevated glucose concentration is also an effect of the hyperactivity of some independent metabolic pathways such as monosaccharide autooxidation, the extent of non-enzymatic glycosylation, $\mathrm{PKC}$ activation and phospholipase $\mathrm{A}_{2}$, and hyperactivity of the intracellular polyol pathway [24, 28].

The observed disorders in the antioxidant protective system of erythrocytes of patients with type 2 diabetes may indicate a higher susceptibility of "diabetic" erythrocytes to oxidative stress. These disorders are greater with metabolically unbalanced diabetes, which found confirmation in our studies. The elevated concentration of glycolated hemoglobin in diabetes, especially when the ailment is improperly controlled, may not only evoke an increase in oxidative stress, but it may also effect oxidative erythrocyte damage and the rheological changes that go with it, which may then lead to the development of vascular complications in diabetes.

An increased concentration of total cholesterol and triglycerides may have an influence on lowering the activity of the antioxidative system in patients with metabolically unbalanced type 2 diabetes; however, the differences in the values of these parameters in the two groups of diabetic patients were not statistically significant. The mechanism and relationships in the oxidativeantioxidative system of this group of examined patients would perhaps have been more comprehensible if the lipid peroxidation products or the ATP concentration in erythrocytes had been determined, because one of the after-effects of intensified exposure of cells to oxidative stress is lowered ATP level; however, this requires further and broader studies [31].

In conclusion, the obtained results confirm the thesis of glucose toxicity and intensification of oxidative stress in diabetes. Long-term hyperglycemia leads, through the activation of various metabolic pathways, to an intensification of free radical processes and a weakening of antioxidative mechanisms.

\section{REFERENCES}

1. Aydin A., Orhan H., Sayal A., Ozata M., Sahin G. and Isimer A. (2001): Oxidative stress and nitric oxide parameters in type II diabetes mellitus: effects of glycemic control. Clin. Biochem., 34, 65-70.

2. Banach M., Markuszewski L., Zaslonka J., Grzegorczyk J., Okonski P. and Jegier B (2004): The role of inflammation in the pathogenesis of atherosclerosis. Przegl. Epidemiol., 58, 663-670.

3. Bartosz G. (1996): Peroxynitrite: mediator of the toxic action of nitric oxide. Acta Biochem. Pol., 43, 646-659.

4. Baynes J. W. (1991): Role of oxidative stress in development of complications in diabetes. Diabetes, 40, 405-412.

5. Baynes J. W. and Thorpe S. E. (1999): Role of oxidative stress in diabetic complications: a new perspective on an old paradigm. Diabetes, 48, 1-9.

6. Beers R. F. Jr. and Sizer I. W. (1952): Spectrophotometric method for measuring the break down of hydrogen peroxide by catalaze. J. Biol. Chem., 195, 133-140.

7. Boel E., Selmer J., Flodgaard H. J. and Jensen T. (1995): Diabetic late complications; will aldose reductase inhibitors or inhibitors of advanced glycosylation endproducts formation hold promise? J. Diabetes Complications, 9, 104-129.

8. Bono A., Caimi G., Catania A., Sarno A. and Pandolfo L. (1987): Red cell peroxide metabolism in diabetes mellitus. Horm. Metab. Res., 19, 264-266.

9. Brownlee M. (1994): Glycation and diabetic complications. Diabetes, 43, 836-841.

10. Catherwood M. A., Powell L. A., Anderson P., McMaster D., Sharpe P. C. and Trimble E. R. (2002): Glucose-induced oxidative stress in mesangial cells. Kidney Int., 61, 599-608.

11. Cederberg J., Basu S. and Eriksson U. J. (2001): Increased rate lipid peroxidation and protein carbonylation in experimental diabetic pregnancy. Diabetologia, 44, 766-774.

12. Ceriello A. (1997): Acute hyperglycaemia and active stress generations. Diabet. Med., 14 (suppl. 13), 45-49.

13. Ceriello A., Giugliano D., Quatraro A., Donzella C., Dipalo G. and Lefebvre P. J. (1991): Vitamin E reduction of protein glycosylation in diabetes. New prospect for pre- 
vention of diabetic complications? Diabetes Care, 14, 68-72.

14. Cheeseman K. H. (1993): Mechanisms and effects of lipid peroxidation. Mol. Aspects Med., 14, 191-197.

15. Cheeseman K. H and Slater T. F. (1993): An introduction to free radical biochemistry. Br. Med. Bull., 49, 481-493.

16. Crouch R., Kimsey G., Priest D. G., Sarda A. and Buse M. G. (1978): Effect of streptozotocin on erythrocyte and retinal superoxide dismutase. Diabetologia, 15, 53-57.

17. DeLange R. J. and Glazer A. N. (1989): Phycorerythrin fluorescence-based assay for peroxy radicals: a screen for biologically relevant protective agents. Anal. Biochem., 177, 300-306.

18. Edelman S. V. (1998): Type II diabetes mellitus. Adv. Intern. Med., 43, 449-500.

19. Fu M. X., Knecht K. J., Thorpe S. R., and Baynes J. W. (1992): Role of oxygen in cross-linking and chemical modification or collagen by glucose. Diabetes, 41 (suppl. 2), $42-48$.

20. Godin D. V., Wohaieb S. A., Garnett M. E. and Goumeniuk A. D. (1988): Antioxidant enzyme alterations in experimental and clinical diabetes. Mol. Cell. Biochem., 84, 223-231.

21. Halliwell B. (1990): How characterize a biological antioxidant. Free Radic. Res. Commun., 9, 1-32.

22. Halliwell B., Aeschbach R., Loliger J. and Aruoma O. I. (1995): The characterization of antioxidants. Food Chem. Toxicol., 33, 601-617.

23. Jaeschke H. (1995): Mechanisms of oxidant stress induced acute tissue injury. Proc. Soc. Exp. Biol. Med., 209, 104-111.

24. Kawamura N., Ookawara T., Suzuki K., Konishi K., Mino M. and Taniguchi N. (1992): Increased glycated Cu, Zn-superoxide dismutase levels in erythrocytes of diabetics with insulin-dependent diabetes mellitus. J. Clin. Endocrinol. Metab., 74, 1352-1354.

25. Kedziora J., Blaszczak R., Ertel D., Rutkowski M., Kedziora-Kornatowska K., Kornatowski T., Rysz J., Kujawski K. and Stachura D. (2005): Selected antioxidative enzyme activities in patients with diabetes mellitus type 2. Arch. Med. Sci., 1, 144-147.

26. Kedziora-Kornatowska K. (1999): The pharmacological attempts of the oxidative stress modification in rats' kidney and blood in the course of streptozotocin diabetes. Mil. Med. Survey, 2, 67-72.

27. Kowalski J., Barylski M., Banach M., Grycewicz J., Irzmanski R. and Pawlicki L. (2006): Neutrophil superoxide anion generation during atorvastatin and fluvastatin therapy used in coronary heart disease primary prevention. J. Cardiovasc. Pharmacol., 48, 143-147.
28. Matkovics B., Varga S. I., Szabo L. and Witas H. (1982): The effect of diabetes on the activities of the peroxide metabolism enzymes. Horm. Metab. Res., 14, 77-79.

29. Misra H. P. and Fridovich I. (1972): The role of superoxide anion in the autooxidation of epinephrine a sample assay for superoxide dismutase. J. Biol. Chem., 247, 3170-3175.

30. Pawlak W., Kedziora J., Zolynski K., Kedziora-Kornatowska K., Blaszczyk J., Witkowski P. and Zieleniewski J. (1998): Effect of long term bed rest in men on enzymatic antioxidative defence and lipid peroxidation in erythrocytes. J. Gravit. Physiol., 5, 163-164.

31. Rysz J., Banach M., Stolarek R., Pasnik J., Cialkowska-Rysz A., Koktysz R., Piechota M. and Baj Z. (2007): Serum matrix metalloproteinases MMP-2 and MMP-9 and metalloproteinase tissue inhibitors TIMP-1 and TIMP-2 in diabetic nephropathy. J. Nephrol., 20, 444-452.

32. Rysz J., Banach M., Stolarek R., Paśnik J., CiałkowskaRysz A., Markuszewski L. and Baj Z. (2006): TNF- $\alpha$ priming effect on polymorphonuclear leukocytes reactive oxygen species generation and adhesion molecule expression in hemodialyzed patients. Arch. Immunol. Ther. Exp., 54, 209-215.

33. Rysz J., Blaszczak R., Kedziora-Kornatowska K., Kujawski K., Jablonski S. and Markuszewski L. (2005): Adenosine and neutrophil CD11b/CD62L and oxidative burst in stable ischemic heart disease. Arch. Med. Sci., 1, 94-97.

34. Rysz J., Potargowicz E., Banach M., Łuczyńska M., Stolarek R., Bialasiewicz P., Kasielski M. and Nowak D. (2006): Increased whole blood chemiluminescence in patients with chronic renal failure independent of hemodialysis treatment. Arch. Immunol. Ther. Exp., 54, 347-355.

35. Salahudeen A. K. (1995): Role of lipid peroxidation in $\mathrm{H}_{2} \mathrm{O}_{2}$-induced renal epithelial (LLC-PK1) cell injury. Am. J. Physiol., 268, F30-F38.

36. Salahudeen A. K., Kanji V., Reckelhoff J. F. and Schmidt A. M. (1997): Pathogenesis of diabetic nephropathy: a radical approach. Nephrol. Dial. Transplant., 12, 664-668.

37. Vincent A. M., Brownlee M. and Russell J. W. (2002): Oxidative stress and programmed cell death in diabetic neuropathy. Ann. N.Y. Acad. Sci., 959, 368-383.

38. Zozulinska D. and Wierusz-Wysocka B. (2005): Hyperglycaemia and inflammation are culprits of late diabetic complications. Arch. Med. Sci., 1, 115-118.

Added in proof:

39. Rogowicz A., Litwinowicz M., Piłaciński S., Zozulińska G. and Wierusz-Wysocka B. (2007): Does early insulin treatment decrease the risk of microangiopathy in non-obese adults with diabetes. Arch. Med. Sci., 3, 129-135. 